ESTUDIO PROSPECTIVO OBSERVACIONAL

\title{
Rol del virus sincicial respiratorio en una cohorte de adultos mayores
}

\author{
Recibido: 12/6/19 Aceptado: 12/8/19
}

Bettina Cooke', Mora Obed ${ }^{1}$, Agustín Romandetta ${ }^{1}$, Natalia Spinelli ${ }^{1}$, M. Fernanda Alzogaray ${ }^{1}$, Gustavo Chaparro', Lucía Galeano ${ }^{1}$, Belén Alcorta', Juan Balbuena', Mara Russo², Martín Avaro², Andrea Czech², Estefanía Benedetti², Andrea Pontoriero², Elsa Baumeister², Analía Mykietiuk'

\section{RESUMEN}

El rol de los virus respiratorios distintos de influenza en las infecciones respiratorias agudas en los adultos mayores ha sido probablemente subestimado. En los últimos años, los avances en técnicas moleculares de diagnóstico han hecho posible la identificación rápida del virus sincicial respiratorio humano (HRSV).

Realizamos un estudio prospectivo observacional para evaluar el rol del HRSV en mayores de 65 años que se hospitalizaron por infecciones respiratorias en nuestra institución, ubicada en la ciudad de La Plata, provincia de Buenos Aires. Fueron reclutados 124 pacientes y el HRSV se detectó en 13, influenza B en 9 e influenza A en 8. La presentación clínica más frecuente de los pacientes con HRSV fue el broncoespasmo y afectó principalmente a personas con comorbilidades. HRSV fue responsable de un número importante de internaciones por enfermedad respiratoria aguda en mayores de 65 años en nuestra institución y se asoció a mortalidad elevada (23\%).

Palabras clave: virus sincicial respiratorio humano, adultos mayores, infecciones respiratorias agudas.
${ }^{1}$ Servicio de Infectología, Instituto Médico Platense, La Plata, Buenos Aires, Argentina.

${ }^{2}$ Centro Nacional de Influenza de la OMS, Servicio Virosis Respiratorias, INEI-ANLI C. G. Malbrán.

Autor para la correspondencia: Analía Mykietiuk. Servicio de Infectología Instituto Médico Platense, Boulevard 51 No 315, La Plata (CP 1900), Buenos Aires Argentina.

Correo electrónico: analiamykietiuk@hotmail.com

Ninguno de los autores presenta conflictos de interés. El estudio fue financiado con fondos propios. Los contenidos de este artículo no han sido publicados previamente. 


\section{Introducción}

El virus sincicial respiratorio humano (HRSV) fue identificado por primera vez en 1956 y se trata de un virus ARN de la familia Pneumoviridae. En nuestro país usualmente su circulación comienza en mayo y se superpone con la del virus de influenza durante seis a ocho semanas (1). Las actuales técnicas moleculares de diagnóstico microbiológico son más específicas y rápidas que el cultivo celular y la serología, permitiendo identificarlo como un agente importante en infecciones respiratorias (2). Si bien es un virus que afecta mayoritariamente a niños, especialmente a los menores de seis meses, se ha reconocido como etiología de las infecciones respiratorias en los mayores de 65 años, sobre todo, en personas que presentan comorbilidades o viven en comunidades cerradas $(3,4)$. La inmunidad generada por el HRSV es incompleta y la re-infección ocurre con frecuencia. En adultos se presenta como una infección del tracto respiratorio con fiebre que no supera los $38^{\circ} \mathrm{C}$. Los síntomas de repercusión general son leves, con un predominio de broncoespasmo y tos (1).

En Argentina, durante 2018 fueron estudiadas y notificadas a través del Sistema Nacional de Vigilancia de Laboratorio (SIVILA) 73.745 muestras para virus respiratorios de pacientes pediátricos y adultos, de las cuales 26.318 (35,6\%) fueron positivas. De las muestras positivas, el $66 \%$ correspondieron a (HRSV); el $17,1 \%$ a virus influenza; $8,9 \%$ a virus parainfluenza; $7,4 \%$ a adenovirus y $4,4 \%$ a metapneumovirus. El $65 \%$ de las muestras estudiadas provenían de pacientes menores de 2 años y el 4,3\% de mayores de 65 años (5). Existe un sesgo pediátrico en la valoración de los virus respiratorios distintos de influenza como agentes etiológicos de infecciones respiratorias y probablemente por ello los estudios prospectivos en adultos son escasos.

El crecimiento de la población de adultos mayores plantea la necesidad de conocer más sobre la etiología y comportamiento clínico de las infecciones que la afectan.

El objetivo de este estudio fue analizar la etiología, características epidemiológicas y clínicas en una cohorte de pacientes mayores de 65 años que se hospitalizaron por infecciones respiratorias agudas en nuestra institución.

\section{Materiales y métodos}

Estudio prospectivo observacional que se llevó a cabo entre el 1 de abril de 2018 y el 1 de febrero de 2019 en un hospital privado de 137 camas en la ciudad de La Plata, provincia de Buenos Aires, Argentina. Se realizó un seguimiento prospectivo de los pacientes que ingresaron con infección respiratoria aguda (IRA) que se internaron en nuestra institución.

Criterios de inclusión: edad 65 años o más, hospitalización, IRA, firma del consentimiento informado. Criterios de exclusión: paciente incapaz o no dispuesto a cumplir con los procedimientos del estudio, hospitalización mayor a 48 horas.

\section{Definiciones}

IRA: infección respiratoria aguda que requiere hospitalización con o sin fiebre.

Neumonía adquirida en la comunidad (NAC): comienzo agudo de al menos dos de los siguientes síntomas: tos, producción de esputo purulento, disnea y/o dolor torácico; más dos de los siguientes signos: fiebre, hipotermia, taquicardia $\mathrm{y} / \mathrm{o}$ taquipnea. Radiología: presencia de infiltrados pulmonares nuevos.

Bronquitis aguda: tos de aparición aguda, habitualmente productiva, que dura más de cinco días (de una a tres semanas) con ausencia de semiología de condensación pulmonar, que puede estar asociada a disnea, dolor torácico, fiebre y malestar general. Radiología: ausencia de infiltrados pulmonares nuevos.

Enfermedad pulmonarobstructiva crónica(EPOC) reagudizada: exacerbación aguda de la situación clínica basal del paciente EPOC con aumento de disnea, tos y expectoración, esputo purulento o combinación de estos tres síntomas.

Exacerbación asmática: episodios caracterizados por aumento de disnea, sibilancias, tos y dolor torácico o una combinación de estos síntomas que conllevan un empeoramiento de la función pulmonar con respecto al estado habitual del paciente.

Neumonía asociada a los cuidados de la salud: neumonía que ocurre en pacientes con alguno de los siguientes criterios: antecedente de hospitalización de al menos dos días dentro 
de los 90 días previos al ingreso hospitalario, residencia en hogares geriátricos, internación domiciliaria, hospital de día y/o hemodiálisis crónica.

Enfermedad tipo influenza (ETI): presentación súbita de fiebre $>38^{\circ} \mathrm{C}$ y tos u odinofagia (en ausencia de otro diagnóstico) acompañado o no de: astenia, mialgias o postración, náuseas o vómitos, rinorrea, conjuntivitis, adenopatías o diarrea.

Mortalidad precoz: mortalidad a las 48 horas del ingreso.

Mortalidad global: mortalidad a los 30 días del ingreso.

Los datos de epidemiología, presentación y evolución clínica fueron recolectados en una tabla ad hoc a través del interrogatorio, examen físico, evaluación de imágenes y estudios de laboratorio. Las muestras microbiológicas obtenidas por protocolo consistían en dos hemocultivos, una muestra de esputo cuando era posible e hisopado nasofaríngeo (HNF) en todos los pacientes enrolados. Las muestras de sangre y esputo fueron procesadas según procedimientos del Clinical and Laboratory Standard Investigation (CLSI). En los HNF se realizó extracción total de ácidos nucleicos utilizando el Mini Kit QIAamp ${ }^{\circledR}$ Viral RNA (QIAGEN), siguiendo las instrucciones del fabricante.

El diagnóstico de virus respiratorios fue mediante la búsqueda de virus sincicial respiratorio (HRSV), metapneumovirus humano, influenza A y B en todas las muestras mediante procedimientos de RT-PCR en tiempo real (qRT-PCR) empleando el kit SuperScript III PlatinumOne-StepqRT-PCR (Thermo Fisher), sondas de hidrólisis con marca dual FAMBHQ1 y primers específicos, procedimientos recomendados por los Centros para el Control y la Prevención de Enfermedades (CDC), de Estados Unidos, para el diagnóstico de los virus mencionados. Para la interpretación de los resultados se siguieron las recomendaciones del CDC (6).

En los casos en que se identificó virus influenza $A$ se realizó luego la determinación del subtipo A(H3N2)yA(H1N1)pdm09 utilizando el CDC Influenza Virus Real-time RT-PCR Panel Influenza A ( $\mathrm{H} 1 / \mathrm{H} 3 / \mathrm{H} 1$ pdm09) Subtyping Panel, catálogo \#FluRUO-04. Todos los protocolos rRT-PCR, cebadores y sondas son propiedad de y fueron proporcionadas por la División de Influenza (DI) del CDC, protocolos disponibles a petición, www.cdc.gov/CLSIS.

La determinación del tipo A y B de HRSV se llevó a cabo mediante un procedimiento de RT-PCR en tiempo real (7).
Las visitas de seguimiento clínico se realizaron al día 3-5 y al día 30 (en forma presencial o telefónica). En la primera visita se evaluaron días de fiebre, complicaciones y mortalidad; al día 30 mortalidad.

\section{Resultados}

Fueron evaluados 298 pacientes, de los cuales 124 cumplieron con todos los criterios de inclusión y ninguno de exclusión. La Tabla 1 muestra las características relevantes de la población analizada. La edad promedio fue 81 años, con un rango de 65 a 98 años, y el $58 \%$ de los pacientes fueron varones. Los diagnósticos clínicos al momento de la admisión fueron neumonía adquirida de la comunidad 90 (72\%), exacerbación de EPOC 11 (9\%), bronquitis aguda 10 (8\%), neumonía asociada a los cuidados de la salud 8 (7\%) y ETI 5 (4\%). En 92 casos se tomaron hemocultivos: 4 pacientes presentaron resultados positivos para Streptococcus pneumoniae, 3 para Escherichia coli, 2 para Staphylococcus aureus meticilino sensible y 1 para Klebsiella pneumoniae, el resto resultaron negativos. Se obtuvieron muestras representativas de esputo en 20 pacientes, sin aislamiento microbiológico.

Se realizó HNF a 124 pacientes. Del total de los hisopados, 30 fueron positivos para virus respiratorios (24\%).

Se identificó HRSV en 13 muestras (43\%), influenza B en 9 (30\%), influenza $A$ en $8(27 \%)$. No se observaron coinfecciones (Figura 1). De los 8 virus influenza $A$ detectados, 5 correspondieron al subtipo A (H1N1) y 3 no pudieron subtipificarse debido a que las muestras mostraron baja carga viral. El $85 \%$ de los HRSV identificados estuvieron asociados al tipo B. El tipo de HRSV y subtipo de Influenza A predominantes en la población estudiada coincidieron con los hallazgos de la Vigilancia Nacional de Virus respiratorios en nuestro país (8).

En referencia a los 13 pacientes positivos para HRSV la edad promedio fue 87 años, $69 \%$ fueron mujeres. El $30 \%$ presentaba antecedentes de insuficiencia cardíaca y $30 \%$ enfermedad respiratoria crónica. El $46 \%$ estaba vacunado para influenza y el 38\% para neumococo. El diagnóstico clínico al ingreso fue NAC en 10 pacientes (77\%), exacerbación de EPOC en 2 (15\%) y bronquitis en un caso (8\%). La presentación clínica en el $85 \%$ de los casos fue broncoespasmo y el $54 \%$ evidenció tos productiva. Nueve pacientes presentaron fiebre, con un rango de duración de entre 1 y 2 días. No se detectaron coinfecciones por virus ni bacterias. La media de estadía hospitalaria fue 9 
Tabla 1. Características de los 124 pacientes que ingresaron con diagnóstico de infección respiratoria aguda

\begin{tabular}{|c|c|}
\hline & $N=124(\%)$ \\
\hline $\begin{array}{l}\text { Edad, años (media } \pm D S \text { ) } \\
\text { Sexo }\end{array}$ & $81 \pm 8,67$ \\
\hline Masculino & $72(58)$ \\
\hline Femenino & $52(42)$ \\
\hline \multicolumn{2}{|l|}{ Comorbilidades } \\
\hline EPOC & $26(20)$ \\
\hline Diabetes & $22(17)$ \\
\hline Tabaquismo & $16(12)$ \\
\hline Neoplasia & $13(10)$ \\
\hline Enfermedad cerebrovascular & $10(8)$ \\
\hline Asma & $6(4)$ \\
\hline Obesidad & $4(3)$ \\
\hline Inmunocompromiso & $5(4)$ \\
\hline Demencia & $5(4)$ \\
\hline Enfermedad neurológica & $5(4)$ \\
\hline Insuficiencia renal crónica & $6(5)$ \\
\hline Insuficiencia cardíaca congestiva & $1(0.8)$ \\
\hline Hepatopatía & $1(0.8)$ \\
\hline Vacunación antigripal & $39(31)$ \\
\hline Vacunación antineumocócica & 24 (19) \\
\hline \multicolumn{2}{|l|}{ Presentación clínica } \\
\hline Neumonía adquirida de la comunidad & $90(72)$ \\
\hline EPOC reagudizado/ASMA & $11(9)$ \\
\hline Bronquitis & $10(8)$ \\
\hline Neumonía asociada ámbito sanitario & $8(7)$ \\
\hline Gripe & $5(4)$ \\
\hline Días de tratamiento antibiótico (media \pm DS) & $8,36 \pm 5,7$ \\
\hline Días de internación promedio (media $\pm D S$ ) & $9 \pm 13.5$ \\
\hline Mortalidad precoz * & $2(1)$ \\
\hline Mortalidad globalı & $38(30)$ \\
\hline
\end{tabular}

*Mortalidad precoz: mortalidad a las 48 horas del ingreso. \pm Mortalidad global: mortalidad a los 30 días del ingreso.
Tabla 2. Características clínicas y de laboratorio de los 13 pacientes con diagnóstico de IRA por VSR

\begin{tabular}{|l|l|}
\hline & $\mathbf{N}=\mathbf{1 3}(\%)$ \\
\hline Fiebre* & $\mathbf{9}(\mathbf{7 0})$ \\
\hline $\begin{array}{l}\text { Días de fiebre (media } \pm \text { DS) } \\
\text { Broncoespasmo }\end{array}$ & $\mathbf{1 \pm 0 , 8}$ \\
\hline Rales crepitantes & $11(85)$ \\
\hline Tos con expectoración & $8(62)$ \\
\hline Saturación de $02 \leq \mathbf{9 4 \%}$ & $\mathbf{7 ( 5 4 )}$ \\
\hline Leucocitosis $\geq 10$ mil/mm \\
Comorbilidades & $\mathbf{6}(\mathbf{4 6})$ \\
\hline EPOC & $\mathbf{5}(\mathbf{4 0})$ \\
\hline Tabaquismo & \\
\hline Asma & $1(7,7)$ \\
\hline Demencia & $1(7,7)$ \\
\hline Insuficiencia renal crónica & $3(23)$ \\
\hline Insuficiencia cardíaca congestiva & $1(7,7)$ \\
\hline Días de hospitalización & $1(7,7)$ \\
\hline Mortalidad & $3(23)$ \\
\hline
\end{tabular}

*Fiebre: temperatura mayor o igual a $38^{\circ} \mathrm{C}$.

\section{Figura 1. Etiologías virales}

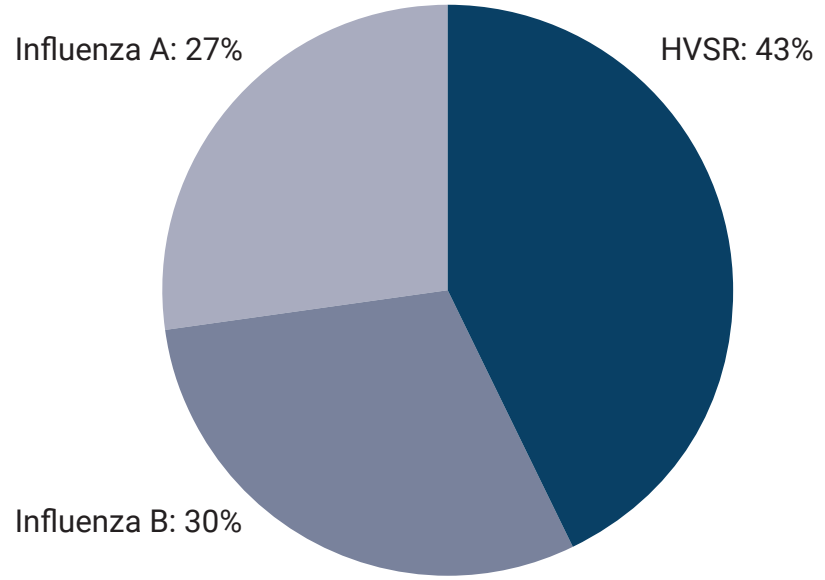

Distribución proporcional de virus respiratorios identificados. N:30 
días y el rango fue 4 a 16 días. La duración del tratamiento antibiótico promedio fue 9,5 días y el rango fue 4 a 15 días. No se observó mortalidad precoz y la mortalidad global fue del 23\% (3 pacientes). Los tres pacientes fallecieron durante la internación y su muerte fue relacionada clínicamente a la infección por HRSV. Dos de los tres pacientes fallecidos presentaban dos o más comorbilidades. En la Tabla 2 se describen los signos y síntomas y los hallazgos de laboratorio de los pacientes con diagnóstico de HRSV.

\section{Discusión}

El rol de HRSV como agente responsable de infecciones severas en adultos es subestimado debido al enfoque pediátrico de esta infección. Si bien disponemos de datos epidemiológicos nacionales a través del SIVILA, no existen publicaciones sobre datos clínicos en adultos mayores.

Una de las causas puede ser lo dificultoso de su diagnóstico. El diagnóstico de esta infección en adultos a través de los métodos tradicionales (cultivo celular y serología) no es útil en el escenario agudo, permitiendo únicamente diagnósticos retrospectivos (9). Los métodos rápidos de detección antigénica demostraron ser sensibles en población pediátrica (80-95\%). En cambio, su sensibilidad en adultos es mucho menor (23\%), quizás por sequedad mucosa, menor carga viral y tiempo de excreción viral más corto (10).

La disponibilidad de técnicas de diagnóstico molecular como la rt-PCR han permitido obtener más información sobre el comportamiento del HRSV. Sin embargo, estos métodos diagnósticos aún no están disponibles masivamente y pertenecen al grupo de técnicas de alto costo $(11,12$ y 13$)$. La alta sensibilidad de estas técnicas de diagnóstico molecular, además de brindar información epidemiológica, podría modificar el manejo de los pacientes tanto desde el punto de vista clínico como en el control de infecciones (9).

El HRSV fue responsable de un número importante de internaciones por enfermedad respiratoria aguda en mayores de 65 años en nuestra institución. Afectó principalmente a personas con comorbilidades, observándose una alta mortalidad en esta población.

El tratamiento disponible para esta patología actualmente está limitado al uso de rivabirina en pacientes inmunocomprometidos debido a su limitada eficacia y alto costo. El palivizumab se ha convertido en el estándar para la prevención de la infección por HRSV en niños de alto riesgo, pero aún no ha sido testeado en adultos mayores (14). Se están realizando diferentes estudios para el desarrollo de vacunas que se muestran promisorios, pero se encuentran en etapas iniciales $(11,12,13)$. Actualmente, las medidas de soporte y la prevención con precauciones de aislamiento respiratorio y de contacto son las únicas herramientas con las que contamos contra esta enfermedad $(18,19,20)$.

La fortaleza de nuestro estudio se encuentra en la homogeneidad de la población analizada, ya que todos los pacientes mayores de 65 años que se hospitalizaban con diagnóstico de infección respiratoria aguda fueron hisopados para detección de virus respiratorios. La debilidad de nuestro análisis es la ausencia de diagnóstico serológico para bacterias intracelulares responsables de cuadros respiratorios, lo que podría subestimar el número de coinfecciones. Otros límites para una conclusión más contundente son el número de pacientes incluidos y el hecho de que el reclutamiento sea en un único centro.

Este estudio confirma que el HRSV es un patógeno relevante en este grupo, asociado a morbilidad y mortalidad significativas. Son necesarios estudios multicéntricos que involucren mayor número de pacientes no solo para poder conocer el impacto de este patógeno en la creciente población de adultos mayores, sino también para evaluar si la incorporación de técnicas moleculares en la práctica clínica modifica el pronóstico de la enfermedad. 


\section{Referencias}

1. Edward E. Walsh. Respiratory Syncytial Virus infection: an illness for all ages Clin Chest Med. 2017 March; 38(1): 29-36.

2. Galván JM, Rajas 0 , Aspa J. Review of Non-bacterial Infections in Respiratory Medicine: Viral Pneumonia. Arch Bronconeumol. 2015 Nov; 51(11):590-7.

3. Ann R. Falsey, M D, Patricia A. Hennessey, R N, Maria A. Formica, M S, Crishtopher Cox, Ph D., and Eduard E. Walsh, M D. Respiratory syncytial virus infection in elderly and high risk adults. N Engl J Med 2005; 352(17):174959.

4. Lucion M F, Juárez Medel V, Viegas M, Castellano V, Romaning V S, Grobaporto M, et al. Respiratory Syncytial virus: clinical and epidemiological pattern in pediatric patients admitted to a children's hospital between 2000 and 2013. Arch Argent Pediatr 2014 Oct; 112(5):397-404.

5. Boletín integrado de vigilancia 2018 N 433 SE 51 (acceso: 28 de marzo de 2019)https://www.argentina.gob.ar/ sites/default/files/biv_433_se_51.pdf.

6. Kim C, Ahmed JA, Eidex R B, Nyoka R, Waiboci L W, et al (2011) Comparison of Nasopharyngeal and Oropharyngeal Swabs Respiratory Viruses by Real-Time Reverse Transcription- PCR Assays. PLoS ONE 6(6): e21610.doi: 10.1371/journal.pone.0021610.

7. Hu A, Colella M, Tam J, Rappaport R and Cheng S: Simultaneous detection, Subgrouping and Quantitation of Respiratory Sincitial Virus A and B by Real- Time PCR. J CLIN MICROBIOL, Jan. 2003, 41 (1): p 149-154.

8. Ministerio de Salud de la Nación, Argentina. Boletín Integrado de Vigilancia $N^{a}$ 436, Semana Epidemiológica 03:- 2019 pg 38 : Informe especial del Laboratorio Nacional de Referencia, INEI-ANLIS-Carlos Malbrán

9. H.-S. Huang, C.-L. Tsai, J. Chang, T.-C. Hsu, S. Lin, C.-C. Lee: Multiplex PCR system for the rapid diagnosis of respiratory viruses infection: systematic review and meta- analysis. Clinical Microbiology and Infection 2018 oct; 10: 1055-1063

10. Casiano Colon, A E, Hulbert, B B, Mayer, T K, Walsh, E E, Falsey, A R Lack of sensitivity of rapid antigen tests for the diagnosis of respiratory syncytial virus infections in adults Journal of Clinical Virology 2003; 28:169-174

11. Centros para el Control y la Prevención de Enfermedades. Pruebas de diagnóstico rápido de influenza: información para directores de laboratorios (acceso: 29 de marzo de 2019). https://espanol.cdc.gov/enes/flu/professionals/ diagnosis/rapidlab.htm.

12. Carballal, G Cap. 13: Virus Respiratorios. En Virología Médica, Caraball G y Oubiña J 2015. Editorial Corpus
ISBN 978987-1860-104

13. Videla C. Diagnóstico de infecciones virales en pacientes oncológicos: virus respiratorios, herpes simple, varicela y citomegalovirus. Guías de recomendaciones sobre diagnóstico, tratamiento y prevención de infecciones en pacientes con cáncer 2013 Consenso de la Sociedad Argentina de Infectología. Revista Argentina de Microbiología 2014; 46(Supl.1): 26-36

14. Sethi S, Murphy TF. RSV infection-not for kids only. NEJM. 2005; 352:1810-1812.

15. Clark, CM, Guerrero-Plata. Respiratory Syncytial Virus Vaccine Approaches: a Current Overview. A. Curr Clin Micro Rev (2017) 4: 202-207.

16. Shaw C A, Ciarlet M, Cooper, B W, Dionigi L, Keith P, O’Brien $K B$, et al. The path to an RSV vaccine. Current Opinion in Virology. 2013, 3:332-342.

17. Anderson L J, Dormitzer P R, Nokes D J, Rappuoli R, Roca A, Graham B S. Strategic priorities for respiratory syncytial virus (RSV) vaccine development. Vaccine 31S (2013) B209-B2015.

18. French C E, McKenzie B C, Coope C, Rajanaidu S, Paranthaman K, Pebody R, et al. Risk of nosocomial respiratory syncytial virus infection and effectiveness of control measures to prevent transmission events: a systematic review. Clin Chest Med. 2016 Jul; 10(4):26890.

19. Lehners N, Tabatabai J, Prifert C, Wedde M, PuthenparambilJ, WeissbrichB, etal. Long TermShedding of Influenza Virus, Parainfluenza Virus, Respiratory Syncytial Virus and Nosocomial Epidemiology in Patients with Hematological Disorders. PLOS ONE. February 11, 2016. 11 (2): e0148258.

20. Public Health England Infection control precautions to minimise transmission of acute respiratory tract infections in health care settings. Versión 2. Octubre 2016. (acceso 29 marzo 2019). Disponible en: https:// assets.publishing.service.gov.uk/government/uploads/ system/uploads/attachment_data/file/585584/RTI_ infection_control_guidance.pdf. Acceso 29 de marzo 2019. 
Respiratory Syncytial Virus in the elderly adults

\section{Summary}

The role of respiratory viruses other than influenza in acute respiratory tract infections among elderly adults has probably beenunderestimated. Recentadvancesinmoleculardiagnosis have made the rapid identification of human respiratory syncitial virus HRSV infection possible. We conducted a prospective observational study to evaluate the role of HRSV in elderly patients ( $>65$ years of age) hospitalized for acute respiratory infections. A total of 124 patients were recruited, HRSV infection was identified in 13 patients, Influenza B in 9 patients and influenza $A$ in 8 patients. The most frequent clinical presentation was bronchospasm and the infection was prevalent in patients with comorbidities. HRSV infections accounted for an important number of hospital admissions and has been associated with high mortality rates $(23 \%)$.

Keywords: human respiratory syncytial virus, elderly adults, acute respiratory tract infections. 